\title{
Anti-insulin resistance effects of salidroside through mitochondrial quality control
}

\author{
Baiyang You, Yaoshan Dun, Wenliang Zhang, Lingjun Jiang, Hui Li, Murong Xie, Yuan Liu and Suixin Liu \\ Cardiac Rehabilitation Center, Department of Rehabilitation, Xiangya Hospital of Central South University, Changsha, Hunan, China
}

Correspondence should be addressed to S Liu: liusuixin@csu.edu.cn

\begin{abstract}
Mitochondrial quality control (MQC) and function are determinants for cellular energy metabolism, and their disorders are reported to play an important role in the development of insulin resistance (IR). Salidroside was reported to have beneficial effects on MQC through AMPK pathway; however, it is unknown whether salidroside exerts anti-IR effect with this action. This study sought to investigate the effects of salidroside on IR with an exploration of the mechanisms of its action. Experimental IR models were adopted in high-fat-diet (HFD)-fed mice and palmitate-treated C2C12 myotubes, respectively. Blood levels of glucose and insulin as well as cellular glucose uptake were determined, and mitochondrial function and MQC-associated parameters and reactive oxygen species (ROS) production were analyzed based on treatments with the activator (AICAR), inhibitors (compound C and EX-527) or specific siRNA of Ampk/Sirt1 and mitochondrial ROS scavenger (mito-TEMPO). Protein expression level was determined by Western blot, cellular observation by transmission electron microscope and ROS production by functional analysis kits. Salidroside reduced IR and activated insulin signaling along with the stimulation of AMPK/SIRT1 signaling and downstream regulation of MQC and ROS production. These salidroside effects were comparable to those of AICAR and could be prevented by AMPK/SIRT1 inhibitors or siRNAs, respectively. Salidroside reduces IR and regulates MQC and ROS production by activating AMPK/SIRT1 signaling pathway. Since IR is a critical issue for public health, to explore a potent agent against IR is of high interest. The anti-IR effects of salidroside warrant further experimental and clinical studies.
\end{abstract}

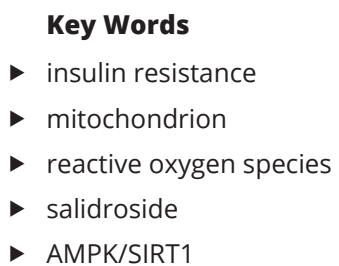

Journal of Endocrinology (2020) 244, 383-393

\section{Introduction}

Insulin resistance (IR) is characterized by the reduction of insulin-stimulated glucose uptake and disposal in insulin target tissues including skeletal muscle, the liver, the heart and the adipose, which could be responsible for the development of type 2 diabetes mellitus (Eckel et al. 2005). Though the underlying mechanisms of IR are not thoroughly understood, a number of factors contributing to IR are well known, for example, excessive energy supply and reduced physical activity (Popkin 2006). In the established experimental animal model, IR can be achieved by fatty acid (FA) through high-fat diet (HFD), while in the cell culture model, IR can be achieved by palmitate, where increased flux of energy fuel took place along with augmented reactive oxygen species (ROS) production and mitochondrial dysfunction, and incomplete lipid oxidation and increased lipid accumulation in skeletal 
muscle are considered as responsible for IR development (Krebs \& Roden 2004, Anderson et al. 2009, Cade 2018).

A previous study has shown that FA-induced mitochondrial dysfunction facilitates pathological ROS production and leads to impaired insulin sensitivity (Rains \& Jain 2011). Then, excessive ROS aggravates mitochondrial dysfunction in the skeletal muscle of an HFD-fed diabetic mouse model, which might exacerbate the impact of the mitochondrial ROS generation and result in a vicious feedforward cycle (Bonnard et al. 2008, Wang et al. 2016). A previous study shows that transgenic mice expressing a mitochondria-targeted form of catalase were resistant to HFD-induced IR, which indicates the involvement of mitochondria-derived ROS in the pathogenesis of IR (Anderson et al. 2009).

Mitochondria are the most important organelle involved in the cellular energy metabolism and the major site of ROS production (Tiganis 2011), and mitochondrial quality control (MQC) consisting of mitochondrial biogenesis, fusion, fission and autophagy serves as the key cellular process to maintain mitochondrial homeostasis (Suliman \& Piantadosi 2016, Xie et al. 2019). Any disturbance of this cellular process can result in mitochondrial dysfunction. A previous study has shown that IR was associated with mitochondrial dysfunction/ limited oxidative capacity (Kim et al. 2008). It has been reported that there was a global expression downregulation of mitochondrial oxidative pathway with concomitant downregulation of mtDNA amount, mtDNA-dependent translation system and protein levels in obese twins compared to the lean co-twins, suggesting a relationship between mitochondrial biogenesis and IR (Yan et al. 2013). A recent study shows that intervention leading to reduced IR was associated with depressed mitophagy (Jung \& Lee 2010, Ren et al. 2019). Furthermore, the treatment leading to IR resulted in distorted mitochondrial fission and fusion (Lin et al. 2018).

In the regulation of MQC there are diverse factors involved in different pathways (Suliman \& Piantadosi 2016). AMPK/SIRT1 (adenosine 5-monophosphateactivated protein kinase/sirtuin 1) is regarded as a cellular energy sensor that is activated by high AMP/ATP ratio and inhibited by excessive energy supply (Gowans \& Hardie 2014). Moreover, AMPK was found to be associated with biomarkers for diabetes (Huang et al. 2013), but the mechanisms are not fully understood. Previous studies have demonstrated that AMPK/SIRT1 plays an important role in maintaining MQC (Herzig \& Shaw 2018), and furthermore their activators (i.e. AMPK activator, AICAR (5-aminoimidazole-4-carboxamide-1-b-D-ribofuranoside), and SIRT1 activator, resveratrol) have a distinct impact on MQC via promoting mitochondrial autophagy and biogenesis (Wang et al. 2018, Zhang et al. 2018), whereas their inhibitors (i.e. AMPK inhibitor, compound C (6-[4-(2-piperidin-1-yl-ethoxy)-phenyl)]-3-pyridin-4yl-pyrrazolo[1,5-a]-pyrimidine), and SIRT1 inhibitor, EX-527 (6-chloro-2,3,4,9-tetrahydro-1H-carbazole-1carboxamide)) block these effects (Yan et al. 2013, Ansari Dezfouli et al. 2019). Impaired MQC is reported to increase ROS production (Lin et al. 2018), which is closely associated with IR (Bonnard et al. 2008). In the modeling of MQC, a series of transcript factors are upstream-regulated by AMPK/SIRT1 and downstream regulates MQC, including microtubule-associated protein 1 light chain 3 (LC3), BCL2/adenovirus E1B $19 \mathrm{kDa}$ protein-interacting protein 3 (BNIP3) (Hamacher-Brady \& Brady 2016), peroxisome proliferator-activated receptor gamma coactivator 1 alpha (PGC1A) (Herzig \& Shaw 2018), dynamin-related protein 1 (DRP1) and mitofusin-1 (MFN1 ) (Chen et al. 2003), are involved in mitophagy, biogenesis and fusion/fission (Tanaka et al. 2010).

In this context, agents that have a beneficial effect on MQC would counteract IR and improve insulin sensitivity (Um et al. 2010). Recent studies have demonstrated that salidroside, a phenylpropanoid glycoside compound, extracted from the roots of Rhodiola rosea, has a protective effect on mitochondrial injury (Cao et al. 2005, Zhang et al. 2015), can attenuate oxidative stress in diabetic mice (Li et al. 2011a) and, in combination with exercise, can improve exercise tolerance and MQC (Dun et al. 2017). However, whether these effects of salidroside would contribute to the reduction of IR and furthermore whether MQC is associated with this action remain to be investigated. Since IR is a critically important issue for the public health, to search a potent agent counteracting IR and to understand its mechanism are of high interest. This study sought to investigate the effects of salidroside on IR and the potential mechanisms.

\section{Materials and methods}

\section{Ethics statement}

All animal procedures were carried out in accordance with the guidelines for the use of live animals of National Institute of Health and were approved by the Medicine Animal Welfare Committee of Xiangya Medical School, Central South University (Changsha, China) (approval ID: SYXK 2015-0017). Efforts were made to minimize animal suffering. 


\section{Animals and dosage information}

Male C57BL/6J mice (8 weeks old) were purchased from the Laboratory Animal Centre, Xiangya Medical School (Changsha, Hunan, China), certification number SCXK 2014-0010. The mice were housed in temperaturecontrolled $\left(22 \pm 2^{\circ} \mathrm{C}\right)$ quarters with a ratio of $12 \mathrm{~h}$ light:12h darkness and free access to water and food.

After a 1-week adaptive feeding, mice were randomly divided into three groups ( $n=8$ for each): normal diet (ND), HFD, and HFD+salidroside (HFD+SAL). The ND contained fat with $18 \%$ of total calories and HFD with $45 \%$ of total calories. In the HFD+SAL group, mice were given salidroside with $100 \mathrm{mg} / \mathrm{kg}$ weight (Sinopharm Chemical Reagent, Shanghai, China), whereas mice in other groups were given normal saline as vehicle control by gavage $(0.1 \mathrm{~mL} / 10 \mathrm{~g}$ weight $)$ every morning between 9:00 and 10:00 h for 8 consecutive weeks as previously described (Zheng et al. 2015, 2018). Body weights were measured once a week. After 8 weeks, fasted $(5 \mathrm{~h})$ blood samples from tail vein were taken and thereafter muscle tissues (from m. gastrocnemius) were attained after mice were euthanized using an intraperitoneal injection of sodium pentobarbital $(150 \mathrm{mg} / \mathrm{kg})$.

\section{Cell culture}

C2C12 mouse myoblasts (Cobioer Biotechnology Ltd, Nanjing, China) were cultured in Dulbecco Modified Eagle Medium (DMEM) supplemented with 10\% fetal bovine serum and penicillin/streptomycin $(5000 \mathrm{U} / 5000 \mu \mathrm{g} / \mathrm{mL}$; Gibco, Grand Island, NY, USA). The cells were seeded in six wells. At a $75 \%$ confluence, cells were transferred to differentiation media (DMEM containing 2\% horse serum, Gibco) for 5 days. In the prior trial to test salidroside toxicity, it was found that with salidroside at $>50 \mu \mathrm{g} / \mathrm{mL}$ the cultured cells did not survive for the whole experimental procedure; therefore, the dosage of salidroside treatment was set within $50 \mu \mathrm{g} / \mathrm{mL}$. The further procedures are described in the following text.

The differentiated myotubes were treated with $50 \mu \mathrm{g} / \mathrm{mL}$ salidroside or $2 \mathrm{mmol} / \mathrm{L}$ AICAR for $24 \mathrm{~h}$ or $50 \mu \mathrm{mol} / \mathrm{L}$ mito-TEMPO (a mitochondrial-targeted ROS scavenger, (2-(2,2,6,6-tetramethylpiperidin-1-oxyl-4-ylamino)-2oxoethyl) triphenylphosphonium chloride monohydrate, Sigma-Aldrich) for $1 \mathrm{~h}$ in the presence of $0.75 \mathrm{mmol} / \mathrm{L}$ palmitate for $24 \mathrm{~h}$, and then cells were harvested. To further examine the role of AMPK/SIRT1, IR myotubes with $50 \mu \mathrm{g} / \mathrm{mL}$ salidroside treatment were pretreated with either specific siRNAs transfection $(80 \mathrm{nmol} / \mathrm{L}$ AmpksiRNAs, Sirt1-siRNAs or negative control siRNA for $24 \mathrm{~h}$ ), inhibitors $(10 \mu \mathrm{mol} / \mathrm{L}$ compound C, EX-527 or vehicle for $1 \mathrm{~h})$ or mitochondrial ROS scavenger $(10 \mu \mathrm{mol} / \mathrm{L}$ mitoTEMPO for $1 \mathrm{~h}$ ), and then the cells were harvested.

\section{siRNA transfection}

Specific and negative control siRNAs were purchased from RiboBio Co. Ltd. (Guangzhou, China). Ampk and Sirt1 genes silencing was performed using mice siRNAs transfection. Ampk- and Sirt1-specific siRNAs and negative control siRNAs $(80 \mathrm{nmol} / \mathrm{L})$ were transfected into cells using the FECTTM CP Transfection Kit (RiboBio, Guangzhou, China) after $48 \mathrm{~h}$ of differentiation. After differentiation, the transfected cells were treated with salidroside $(50 \mu \mathrm{g} / \mathrm{ml})$ for $24 \mathrm{~h}$. And further experiments were performed after that.

\section{HOMA-IR index}

Plasma glucose and insulin levels were determined by glucometer (Accu-Chek Performa, Roche Diagnostics, Indianapolis, USA) and ELISA (CSB-E05071m, Wuhan, Hubei China), respectively. The homeostasis model assessment of insulin resistance (HOMA-IR) index was calculated according to the formula (Matthews et al. 1985): HOMA-IR=fasting glucose $(\mathrm{mmol} / \mathrm{L}) \times$ fasting insulin $(\mathrm{mU} / \mathrm{L}) / 22.5$.

\section{Western blot analysis}

Muscle tissues or cells were lysed in radioimmunoprecipitation assay (RIPA) buffer (Beyotime) containing $1 \mathrm{mmol} / \mathrm{L}$ phenylmethanesulfonyl fluoride (PMSF; Beyotime) on ice to extract protein, and the protein concentration was determined. After SDS-PAGE, the proteins were detected according to the method described previously (Dun et al. 2017). The primary antibodies against the following proteins were used: p-AMPK (Abcam), AMPK, SIRT1, citrate synthase (CS), phosphorylated protein kinase B (p-AKT), AKT, LC3, BNIP3, PGC1A, MFN1, DRP1 and $\beta$-actin (ACTB) (Proteintech, Rosemont, IL, USA). The HRP-labeled goat anti-rabbit IgGs or goat anti-mice IgGs (Proteintech) were used as secondary antibodies. The protein bands were analyzed using a gel documentation system (Bio-Rad). 


\section{Manganese-dependent superoxide dismutase (MnSOD) and malondialdehyde (MDA) assay}

The xanthine oxidase method was used to determine the activity of MnSOD in muscle tissues and cells, according to the manufacturer's instructions (A001-2, Jiancheng Bioengineering Institute, Nanjing, Jiangsu, China). The mitochondria were isolated from muscle tissues and cultured cells according to established protocols (G006, Nanjing Jiancheng Bioengineering Institute), which was described previously (Dun et al. 2017). The thiobarbituric acid method was applied to detect the content of MDA in mitochondria according to the manufacturer's instructions (A003-2, Nanjing Jiancheng Bioengineering Institute).

\section{Glucose uptake assay}

The palmitate-treated cells were stimulated with $30 \mathrm{mU} / \mathrm{mL}$ insulin or vehicle for $1 \mathrm{~h}$. After insulin stimulation, myotubes were incubated with $500 \mu \mathrm{M} 2$-(N-(7-nitrobenz2-oxa-1,3-diazol-4-yl)amino)-2-deoxyglucose (2-NBDG, Invitrogen, Carlsbad, CA, USA) for $60 \mathrm{~min}$ and then washed with PBS three times to remove free 2-NBDG. The fluorescence intensity of cells containing 2-NBDG was measured on a Synergy ${ }^{\mathrm{TM}} 2$ Multi-Mode Microplate Reader (BioTek) with excitation at $485 \mathrm{~nm}$ and emission at $535 \mathrm{~nm}$.

\section{Cell oxygen consumption rate (OCR) measurement}

C2C12 myoblasts were seeded in XF 24-well microplates (Seahorse Bioscience, Billerica, MA, USA) and differentiated. Mitochondrial inhibitors, including $1 \mu \mathrm{mol} / \mathrm{L}$ oligomycin, $1 \mu \mathrm{mol} / \mathrm{L}$ carbonyl cyanide 4-(trifluoromethoxy) phenylhydrazone (FCCP) and $1 \mu \mathrm{mol} / \mathrm{L}$ rotenone/antimycin $\mathrm{A}$, were added to the medium in order. OCR was measured with extracellular flux analysis (Seahorse Biosciences) at an 8-min time interval.

Basal respiration was the baseline oxygen consumption reading before the addition of inhibitors with the subtraction of non-mitochondrial respiration after rotenone/antimycin A was added. Maximal respiration represented the maximum OCR measurement value after FCCP injection with the subtraction of nonmitochondrial respiration. ATP production respiration was the OCR reduction after adding oligomycin, an inhibitor of ATP synthase. Spare respiratory capacity was calculated by maximal respiration subtracting basal respiration.
After detection, cell protein content was calculated, and OCR was adjusted accordingly.

\section{JC-1 assay for mitochondrial membrane potential (MMP)}

The treated myotubes were stained with JC-1 (Yeasen, Shanghai, China) to assess MMP. The quantitative fluorescence was measured on a Synergy ${ }^{\mathrm{TM}} 2$ Multi-Mode Microplate Reader (BioTek) to determine green and red JC-1 fluorescence, respectively. The red/green fluorescence intensity ratio reflects MMP.

\section{Transmission electron microscope (TEM)}

The treated myotubes were washed with PBS. After centrifugated at $1000 \mathrm{~g}$ for $5 \mathrm{~min}$ at $4^{\circ} \mathrm{C}$, the cells were fixed with $2.5 \%$ glutaraldehyde in $0.1 \mathrm{M}$ phosphate buffer and observed under TEM (Tecnai G2 Spirit, FEI, USA) (Dun et al. 2017).

\section{DCF (2,7-dichlorodi-hydrofluorescein diacetate) staining}

DCF staining was performed to assess ROS production of myotubes. The treated myotubes were stained with $100 \mu \mathrm{mol} / \mathrm{L}$ DCF (Yeasen) for $90 \mathrm{~min}$ in the darkness. And the fluorescence intensity of cells containing DCF was measured with a Synergy ${ }^{\mathrm{TM}} 2$ Multi-Mode Microplate Reader (BioTek).

\section{Mitochondrial ROS assessment}

MitoSOX staining was used to assess the mitoROS of myotubes. The treated myotubes were stained with $5 \mu \mathrm{mol} / \mathrm{L}$ mitoSOX for $60 \mathrm{~min}$ and $100 \mathrm{nmol} / \mathrm{L}$ mitoTracker (Yeasen) for $30 \mathrm{~min}$ in the darkness. And the staining images were acquired using a fluorescence microscope (Eclipse, Nikon, Japan).

\section{Mitochondrial quality control assessment}

For MQC assessment protein expression levels for mitophagy (LC3, BNIP3), mitochondrial dynamics (DRP1, MFN1) and biogenesis (PGC1A) were determined using Western blot (see 'HOMA-IR index' section). Furthermore, mitophagy expressed as mitolysosomes was microscopically observed using LysoTracker and mitoTacker (Yeasen) and a laser scanning confocal microscope (LSCM; Olympus). 


\section{Statistical analysis}

The relative fluorescence intensity and protein quantities were pairwise normalized, and the mean of control group was set to 1 to display. Statistical analyses were performed using Prism 6 software (GraphPad, Inc.). The results are expressed as mean \pm s.E.M. For quantitative variables, mean values were compared using an ANOVA followed by the Student-Newman-Keuls test for multiple groups. A statistical significance was considered at $P<0.05$.

\section{Results}

\section{Salidroside effects on IR and insulin signaling}

In ND-fed mice, the body weight increased continuously, but slightly (Fig. 1A). In HFD-fed mice (with or without salidroside), the body weight also showed a continuous increase, but the increase was greater than that in ND mice, such that the body weight in HFD was significantly higher from 1 week on $P<0.05$ compared to that in ND mice. Though the body weight in HFD+SAL mice showed a lighter tendency, the difference in body weight between HFD and HFD +SAL mice was statistically not significant. In comparison with that in ND, the blood glucose level in HFD mice was distinctly increased, and it decreased in HFD+SAL compared to that in HFD, though still significantly higher than that in ND (Fig. 1B). Compared to that in ND, the insulin level in HFD significantly increased, and the lowest level was observed in HFD +SAL (Fig. 1C). Accordingly, HOMA-IR in HFD was significantly higher than that in ND, and with additional salidroside treatment HOMA-IR significantly decreased to the level slightly higher than that in ND (Fig. 1D).

In the cell culture setting, insulin-stimulated glucose uptake in the control cells, and this stimulation was distinctly attenuated in the palmitate-treated cells (Fig. 1E). The attenuated insulin stimulation of glucose uptake induced by palmitate could be significantly counteracted by the treatments with salidroside, AICAR or mitoTEMPO, respectively. In the Fig. 1E, we found that the ratios of p-IRS1/IRS1 as well as p-AKT/AKT were lowered by HFD in comparison with ND, while the treatment with salidroside led to a significant increase in these ratios although they were still lower than those in ND. Similar results can also be derived from the cell culture setting treated with salidroside, AICAR or mito-TEMPO, respectively (Fig. 1G).

\section{Salidroside effects on ROS production and mitochondrial damage}

In the animal experimental setting, MnSOD activity in skeletal muscle was reduced in HFD group compared to that of ND group (Fig. 2A); additional salidroside treatment to HFD led to a significant reverse of MnSOD activity $(P<0.05)$. MDA content in mitochondria was significantly augmented by HFD in comparison with ND $(P<0.01)$, which was attenuated by salidroside treatment (Fig. 2B).

In the cell culture experiment, MnSOD activity was higher in the PA+SAL-treated cells than that in palmitate-treated cells (Fig. 2C), and MDA level in the palmitate-treated cells was significantly reduced
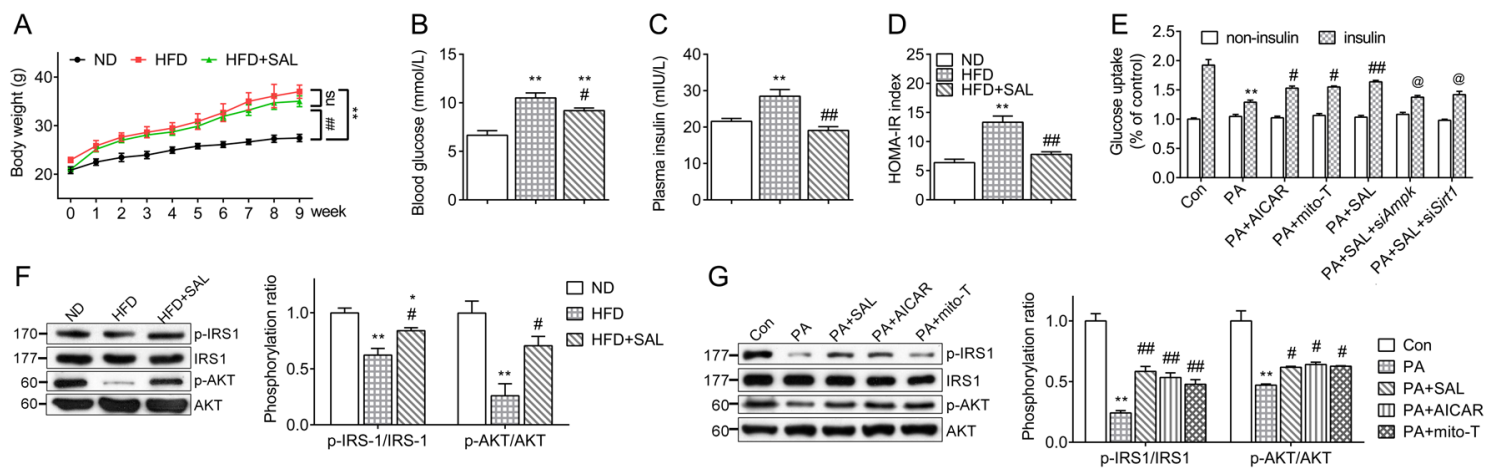

\section{Figure 1}

Salidroside effects on insulin resistance and signaling. Animal experiment (A, B, C, D and F) and cell culture experiment (E and G). ND: normal diet; HFD: high-fat diet; SAL: treatment with salidroside; Con: control without palmitate (PA) treatment; AICAR:5-aminonidazole-4-carboxmide-1-b-D-ribofuranoside; mito-T: mito-TEMPO ((2-(2,2,6,6-tetramethylpiperidin-1-oxyl-4-ylamino)-2-oxoethyl)triphenylphosphonium chloride monohydrate); siAmpk: Ampk-siRNA; siSirt1: Sirt1-siRNA; p-IRS1: phosphorylated insulin substrate 1 (IRS-1); p-AKT: phosphorylated protein kinase B (AKT). ${ }^{*}, *$ represent $P<0.05, P<0.01$ in comparison with ND or Con; \#, \#\# represent $P<0.05, P<0.01$ in comparison with HFD or PA; @, @@ represent $P<0.05, P<0.01$ in comparison with PA + SAL, respectively. 

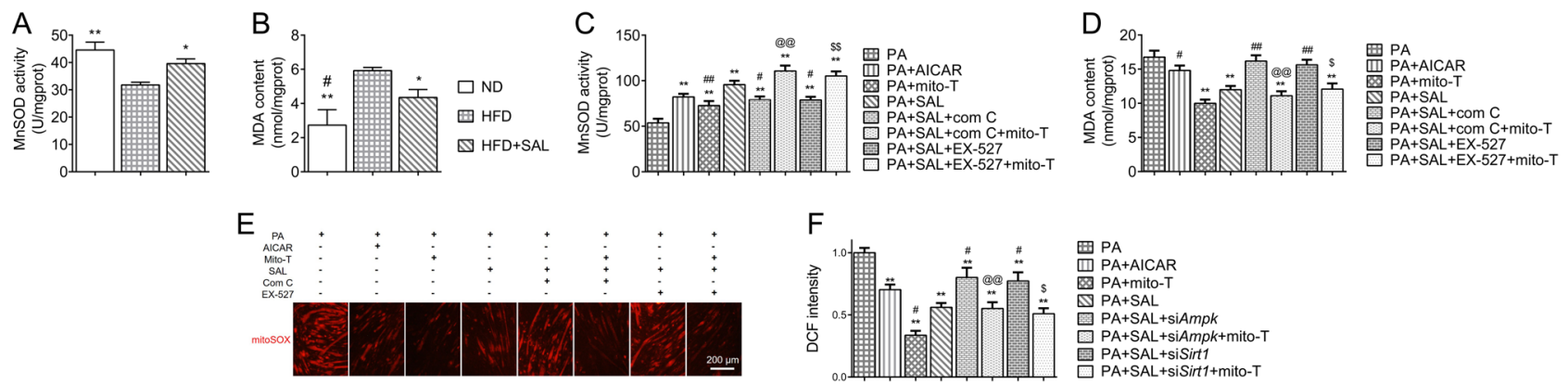

Figure 2

Salidroside effects on ROS production. Animal experiment (A and B) and cell culture (C, D, E, F and G). MnSOD: manganese superoxide dismutase; MDA: malondialdehyde; com C: compound C, 6-[4-(2-piperidin-1-yl-ethoxy-phenyl)]-3-pyridin-4-yl-pyrrazolo[1,5-a]-pyrimidine; EX-527: 6-chloro-2,3,4,9tetrahydro-1H-carbazole-1-carboxamide; mitoSOX: MitoSox staining imaging with a fluorescence microscope (scale bar = $200 \mu \mathrm{m}$ ); DCF: DCFH-DA (2,7-dichlorodi-hydrofluorescein diacetate). For the other abbreviations see legend for Fig. 1. ** represent $P<0.01$ in comparison with PA or HFD; \#, \#\# represent $P<0.05, P<0.01$ in comparison with $\mathrm{PA}+\mathrm{SAL}$; @ represents $P<0.01$ in comparison with $\mathrm{PA}+\mathrm{SAL}+\mathrm{com} \mathrm{C}$ or $\mathrm{PA}+\mathrm{SAL}+\mathrm{si} A m p k$; $\$$, $\$$ represent $P<0.05, P<0.01$ in comparison with $\mathrm{PA}+\mathrm{SAL}+\mathrm{EX}-527$ or $\mathrm{PA}+\mathrm{SAL}+$ siSirt1, respectively.

by additional salidroside treatment (Fig. 2D). The mitochondrial ROS production represented by mitoSOX staining intensity was reduced in PA+SAL-treated cells compared to that from palmitate-treated cells (Fig. $2 \mathrm{E})$. The cellular ROS production revealed through DCF staining intensity is shown in Fig. 2F, where DCF intensity was distinctly lowered by additional salidroside treatment.

In the TEM picture (Fig. 3A), we found that palmitate treatment caused augmented damaged mitochondria with edema and reduction of crest (red arrows) compared to that without palmitate treatment, and the treatment with salidroside could reduce the damaged mitochondria. The basal OCR was comparable between PA group and $\mathrm{PA}+\mathrm{SAL}$ group (ns); however, maximal OCR, spare OCR

A

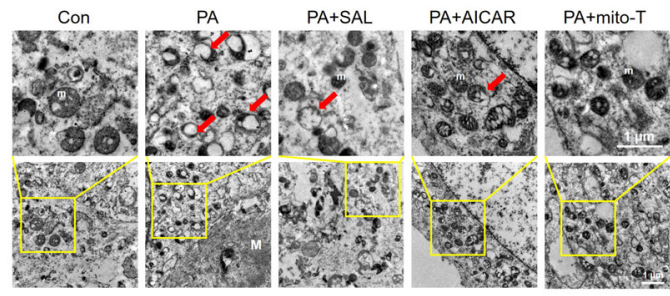

C

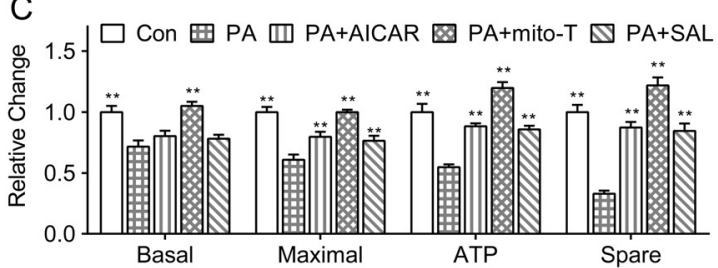

and ATP production were higher in the latter group (Fig. 3B and C). A similar result could be derived for the MMP (Fig. 3D), that is, JC-1 level distinctly higher in the salidroside-treated cells.

\section{Salidroside effects on MQC}

In the cell culture experiment, additional salidroside treatment increased the expression of mitochondrial biogenesis marker PGC1A, mitophagy markers LC3-II and BNIP3 as well as fission/fusion marker DRP1 and MFN1 in palmitate-treated cells (Fig. 4A). The mitochondrial content represented by the protein expression of CS, an essential enzyme during mitochondrial respiration, was augmented in PA+SAL-treated cells compared to that
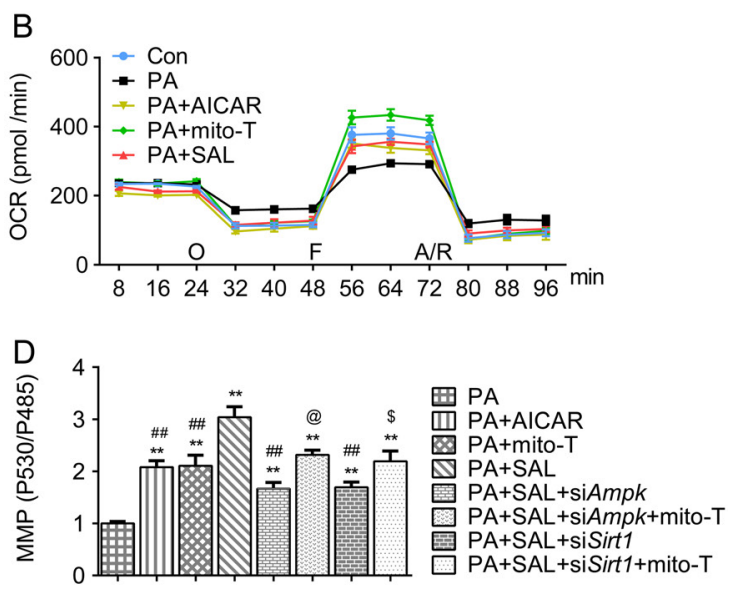

\section{Figure 3}

Salidroside effects on mitochondrial damage. Cell culture experiment. (A), transmission electron microscope imaging; (B and C), OCR: cellular oxygen consumption; (D), mitochondrial membrane potential. The damaged mitochondria were indicated in TEM images (red arrow); m: mitochondria; M: myofilament; for the other abbreviations see legends to Figs 1 and 2. ** represents $P<0.01$ in comparison with $P A$; \#\# represents $P<0.01$ in comparison with PA + SAL; @ represents $P<0.05$ in comparison with PA + SAL + siAmpk; ${ }^{\$}$ represents $P<0.05$ in comparison with $\mathrm{PA}+\mathrm{SAL}+$ siSirt 1 . 
A
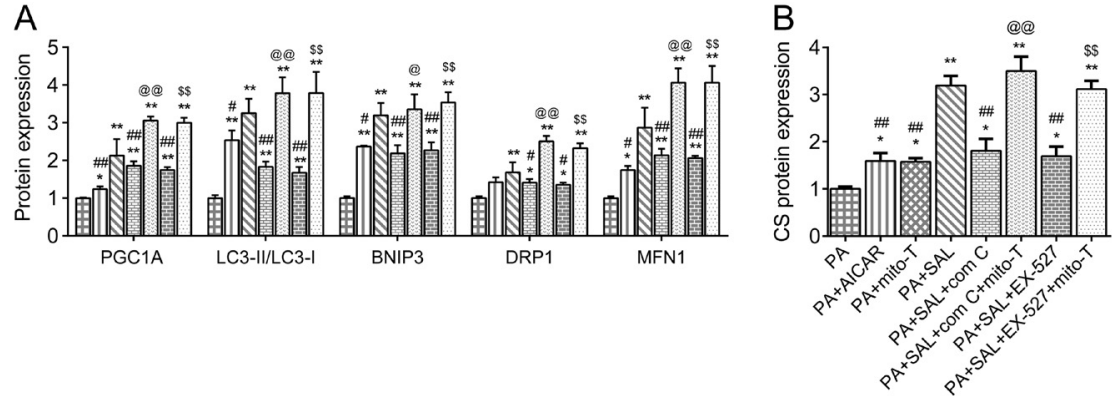
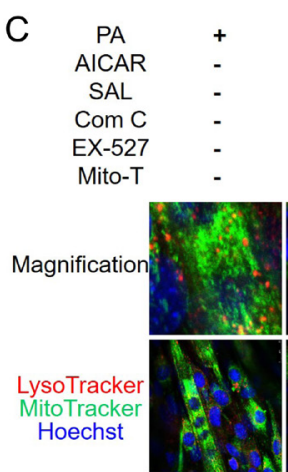
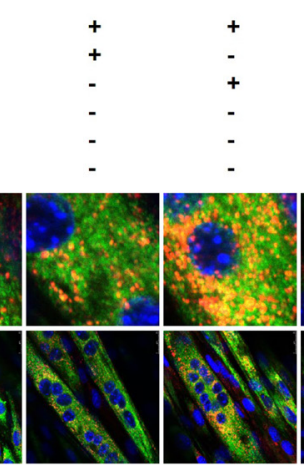
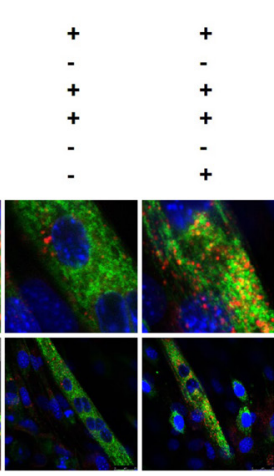

from palmitate-treated cells (Fig. 4B). LysoTraker and MitoTracker were stained in cells, and the colocalization was defined as mitolysosome, which was a marker of mitophagy. As shown in Fig. 4C, mitolysosomes in PA+SAL-treated cells were significantly more abundant than in palmitate-treated cells.

\section{Salidroside exerts anti-IR effect through AMPK/SIRT1 signaling}

In the animal experiment, the p-AMPK and SIRT1 protein expression were reduced in the skeletal muscle of HFD mice when compared to those of ND mice, and additional salidroside treatment to HFD reversed these effects significantly (Fig. 5A). In the cell culture experiment, the AMPK/SIRT1 pathway was activated by salidroside, dependent on dose, where $50 \mu \mathrm{g} / \mathrm{mL}$ was the strongest (Fig. 5B). The treatment with $50 \mu \mathrm{g} / \mathrm{mL}$ salidroside and $2 \mathrm{mmol} / \mathrm{L}$ AICAR significantly elevated the inhibited AMPK/SIRT1 in palmitate-treated myotubes (Fig. 5C).

To examine the role of AMPK/SIRT1 signaling in the effects of salidroside on palmitate-treated cells, AICAR, compound C, EX-527, Ampk-siRNAs, Sirt1 siRNAs and mito-TEMPO were used to treat IR cells. Additional AICAR treatment decreased ROS production (MnSOD, MDA, mito-SOX and DCF) (Fig. 2), facilitated mitochondrial damage (MMP, TEM and OCR) (Fig. 3) and enhanced MQC (protein expression of PGC1A, LC3-II/I, BNIP3, DRP1,

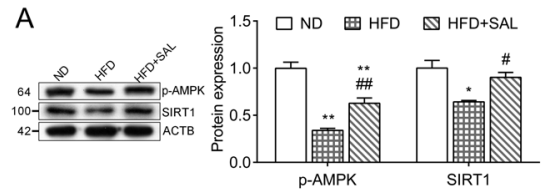

C
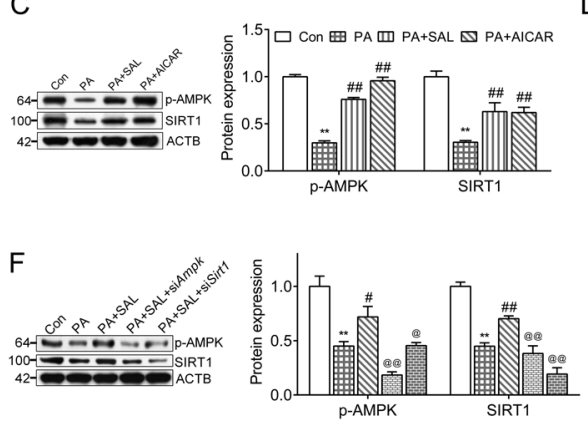
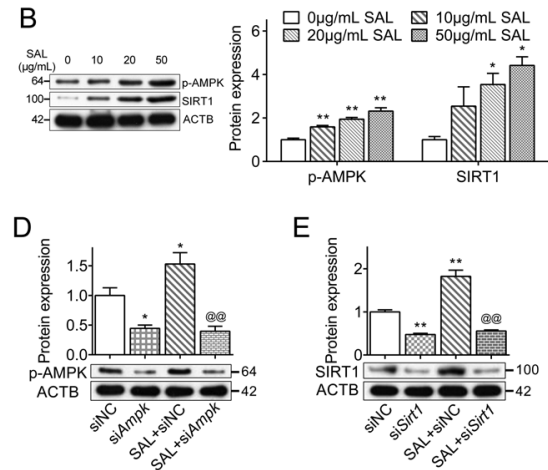

$\square$ Con

田PA

$\triangle P A+S A L$

$P A+S A L+S i A m p k$

国 PA+SAL+SiSirt1

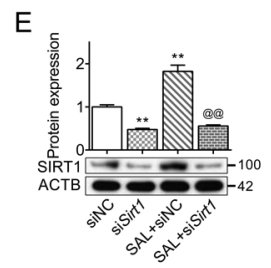

\section{Figure 5}

Salidroside effects on the signaling of mitochondrial quality control. Animal experiment (A) and cell culture experiment (B, C, D, E and F). P-AMPK: phosphorylated AMP-activated protein kinase (AMPK); SIRT1: sirtuin-1; siNC: negative control siRNA; for the other abbreviations see legends to Figs 1 and 2. * ${ }^{(*)} P<0.05(P<0.01)$ in comparison with ND, Con or $P A ; \# P<0.01$ in comparison with HFD. 
MFN1, CS and fluorescence staining of mitolysosomes) (Fig. 4) in palmitate-treated cells. These AICAR effects were comparable to those of salidroside. And the salidroside effects could be inhibited by compound C, EX-527, Ampk and Sirt1 siRNAs. However, mito-TEMPO reversed the effects of AMPK/SIRT1 inhibitors and siRNAs on salidroside-treated cells.

\section{Discussion}

IR is a critical issue for the public health and serves as a central mechanism responsible for the development of diabetes mellitus (Eckel et al. 2005). It is evident that metabolic disorders like excessive fat supply can cause IR (Popkin 2006). In the present study, 8-week HFD in mice led to overweight, hyperglycemia, hyperinsulinemia and, therefore, increased HOMA-IR index compared with ND mice (Fig. 1A, B, C and D). These results suggest the successful establishment of IR model in mice. An additional salidroside treatment to the IR mice, which did not cause any observable change in food intake pattern or gastrointestinal reaction, could, to a greater extent, counteract these results, indicating its anti-IR effects. Furthermore, in the cultured cells palmitate significantly impaired the insulin-stimulated glucose uptake, and salidroside could partly reverse this impairment but did not affect glucose uptake without insulin stimulation (Fig. 1E). Besides these results, we found that the decreased phosphorylation of IRS1 and AKT by HFD in mice (Fig. 1F) or by palmitate in cells (Fig. 1G) could be statistically significantly reversed by salidroside. All these results suggest salidroside effects on glucose metabolism associated with the insulin signaling pathway.

It is evident that in the insulin signaling pathway, ROS plays an important role (Di Meo et al. 2017). An increased ROS production has been shown to impair insulin sensitivity through the inhibition of IRS1 and AKT phosphorylation (Nishikawa et al. 2007, Maurya et al. 2015). In the present study, we showed in Fig. 2A and $\mathrm{C}$ that the decreased activity of MnSOD by HFD or palmitate was distinctly reversed by additional salidroside treatment, and a reciprocal change in mitochondrial MDA could be observed (Fig. 2B and D). Previous studies have shown that an increase in MnSOD activity and a decrease in mitochondrial MDA were closely associated with mitochondrial ROS production (Cheng et al. 2011, Holley et al. 2011). In the present study, we demonstrated that besides excessive mitochondrial ROS production. palmitate impaired mitochondrial function, which is in accordance with the previous study (He et al. 2018). However, these effects could be reversed by additional salidroside treatment (Fig. 2C and D), where the MMP, maximal and spare oxygen consumption and ATP production were elevated compared to those in palmitatetreated cells (Fig. 3B, C and D). Adding mito-TEMPO, a potent antioxidant, into the palmitate-treated cells, the similar effects in glucose metabolism and insulin signaling could be derived (Fig. 1E and G). Accordingly, the treatment intervening mitochondrial ROS production like mito-TEMPO resulted in a remarkable reduction in ROS production (Fig. 2D, E, F and G), along with increased MnSOD activity (Fig. 2C). These data indicate that the regulation of ROS production is involved in the glucose metabolism and insulin sensitivity, and salidroside has an antioxidant effect and this effect is attributed to the reduction of IR. Furthermore, we observed that palmitateinduced mitochondrial dysfunction and damages were attenuated by additional salidroside treatment, and an additional treatment with mito-TEMPO brought about similar results as those of salidroside (Fig. 3). Previous studies demonstrated that the activation of AMPK/SIRT1 axis decreased ROS generation to alleviate oxidative stress (Nemoto et al. 2005, Subauste \& Burant 2007, Wang et al. 2011). Consistent with these findings, our results show that the antioxidant effects of salidroside could be inhibited by AMPK/SIRT1 inhibitors (Fig. 2C, D, E, F and $\mathrm{G})$. These results suggest that salidroside treatment could counteract palmitate-mediated mitochondrial dysfunction and damage, which is associated with the AMPK/SIRT1-modulated ROS production.

It is known that mitochondrial function is determined by MQC processes, including biogenesis, fusion, fission and autophagy (Suliman \& Piantadosi 2016). In the present study we found that CS protein expression level could be remarkably elevated by additional salidroside treatment compared to that in palmitate-treated cells (Fig. 4B), and furthermore, PGC1A was significantly upregulated (Fig. $4 \mathrm{~A})$. Since CS is thought as an indicator of mitochondrial content (Larsen et al. 2012) and PGC1A as a stimulator of mitochondrial biogenesis (Li et al. 2011b), these results suggest that an additional salidroside treatment could enhance mitochondrial biogenesis in palmitate-treated cells. This is further supported by DRP1 and MFN1 data (Fig. 4A), the parameters indicating mitochondrial fission and fusion (Zorzano et al. 2009). Furthermore, LC3 and BNIP were elevated by additional salidroside treatment compared to those in palmitate-treated cells (Fig. 4A), implying that salidroside has impacts on the activity of autophagy (Hamacher-Brady \& Brady 2016), 
which is important for mitochondrial homeostasis (Suliman \& Piantadosi 2016). All these results strongly suggest that salidroside has profound effects on MQC. These effects of salidroside were significantly attenuated by additional treatment with Ampk siRNA or compound C (Fig. 4), suggesting an association between salidroside effects and signaling pathway of AMPK because compound C is regarded as a potent inhibitor of AMPK (Hall et al. 2018). On the other hand, the combination treatment with salidroside and Sirt1 siRNA or EX-527 also led to a similar attenuation of salidroside effects like compound C, suggesting that an association between salidroside effects and signaling pathway of SIRT1 for EX-527 is regarded as a potent inhibitor of SIRT1 (Huang et al. 2017). Moreover, with the combination treatment with salidroside, AMPK suppression and mito-TEMPO in the palmitate-treated cells similar effects to salidroside treatment could be elucidated, and this was also the case for the combination treatment with salidroside, SIRT1 suppression and mito-TEMPO (Figs 2C, D, E, F, 3D and 4A, B, C). These results show that salidroside worked on the signaling pathway upstream to MQC since mito-TEMPO acts as MQC downstream signaling. The data of MQC-related parameters show that salidroside effects were greater than those of AICAR alone, and an additional treatment with AMPK suppression led to an attenuation of salidroside effects but not complete abolishment (Fig. 4). Besides, a combination treatment with salidroside and SIRT1 suppression also attenuated the alone salidroside effects (Fig. 4). These results thus strongly suggest that salidroside effects on IR are attributed to the upstream signaling of MQC, that is, the signaling via AMPK/SIRT1. This seems to be supported by the data from the combination treatment with salidroside, AMPK/SIRT1 inhibitors or siRNA and mito-TEMPO, where mito-TEMPO reversed the inhibitory effects of AMPK/SIRT1 suppression since mito-TEMPO acts on MQC downstream signaling (Lin et al. 2018). All these results strongly suggest that in the action of salidroside effects on IR, the signaling pathway including the activation of AMPK/SIRT1, regulation of MQC, modulation of mitochondrial ROS production and insulin signaling were involved. Unfortunately, we did not perform a combination treatment with salidroside, compound C and EX-527 in palmitate-treated cells to examine whether salidroside effects would be completely abolished by simultaneous blockage of AMPK and SIRT1 pathways. Such a study would help us clarify this point.

From all the results of the present study, we summarized and depicted in Fig. 6 that salidroside stimulated the

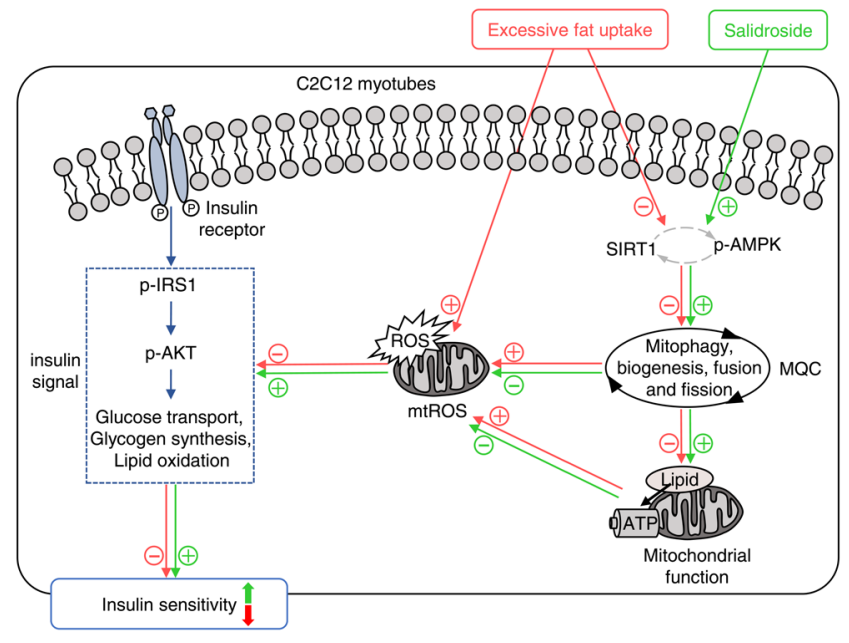

Figure 6

Proposed pathway of salidroside effects on fatty acid-induced insulin resistance. Arrows in green and red show the effects of excessive fat uptake and salidroside on the pointed physiologic processes, respectively; $\oplus$ and $\ominus$ show the facilitating and inhibiting effects, respectively. Fatty acid stimulates mitochondrial ROS production and inhibits the AMPK/SIRT1 pathway in insulin-resistant skeletal muscle, which contributes to the mitochondrial quality control impairment and mitochondrial dysfunction. The destructed mitochondrial homeostasis also leads to excessive ROS production and insulin signaling IRS1/AKT inhibition, which inhibits lipid oxidation, exacerbates lipid accumulation and adversely impacts insulin sensitivity. However, salidroside treatment was found to activate AMPK/SIRT1-mediated mitochondrial quality control, reducing mitochondrial ROS and finally to improve insulin sensitivity.

activation of AMPK/SIRT1 that downstream-regulated MQC, which affected mitochondrial function and ROS production. Finally, the modulation of ROS production exerts a beneficial effect on IR. IR plays a pivotal role in the development of diabetes mellitus and therefore of critical clinical interest for the public health. To explore a potent agent against IR and to understand mechanisms of its action are of high significance. From the present study, we concluded that salidroside has a beneficial effect on IR, which is mediated by the AMPK/SIRT1 via the regulation of MQC and ROS.

\section{Declaration of interest}

The authors declare that there is no conflict of interest that could be perceived as prejudicing the impartiality of the research reported.

\section{Funding}

This work was supported by grants from the National Nature Science Foundation of China (Grant number 81672262 to L S X and 81702241 to $L Y$ ) and the National Development and Reform Commission Foundation of China (No. [2012]1521 to L S X). (c) 2020 Society for Endocrinology Published by Bioscientifica Ltd.
Printed in Great Britain 


\section{Author contribution statement}

$B$ Y, Y D and S L designed experiments; B Y performed the research in cooperation with $L J, M X$ and $\mathrm{H} \mathrm{L}$; $B \mathrm{Y}$ and $\mathrm{W} Z$ interpreted the data and wrote the manuscript; $Y D, S L$ and $Y L$ revised the manuscript.

\section{Acknowledgements}

We acknowledge Xiaoying Wu and Jin Li from the electron microscopy lab of Department of Pathology, Xangya Hospital, for providing experimental instruments and technical assistance. We also acknowledge Wen Meng and Li Hu from the Metabolic Syndrome Research Center of Central South University for providing seahorse XF analyzer and technical assistance.

\section{References}

Anderson EJ, Lustig ME, Boyle KE, Woodlief TL, Kane DA, Lin CT, Price 3rd JW, Kang L, Rabinovitch PS, Szeto HH, et al. 2009 Mitochondrial $\mathrm{H} 2 \mathrm{O} 2$ emission and cellular redox state link excess fat intake to insulin resistance in both rodents and humans. Journal of Clinical Investigation 119 573-581. (https://doi.org/10.1172/JCI37048)

Ansari Dezfouli M, Zahmatkesh M, Farahmandfar M \& Khodagholi F 2019 Melatonin protective effect against amyloid beta-induced neurotoxicity mediated by mitochondrial biogenesis; involvement of hippocampal Sirtuin-1 signaling pathway. Physiology and Behavior 204 65-75. (https://doi.org/10.1016/j.physbeh.2019.02.016)

Bonnard C, Durand A, Peyrol S, Chanseaume E, Chauvin MA, Morio B, Vidal H \& Rieusset J 2008 Mitochondrial dysfunction results from oxidative stress in the skeletal muscle of diet-induced insulin-resistant mice. Journal of Clinical Investigation 118 789-800. (https://doi. org/10.1172/JCI32601)

Cade WT 2018 The manifold role of the mitochondria in skeletal muscle insulin resistance. Current Opinion in Clinical Nutrition and Metabolic Care 21 267-272. (https://doi.org/10.1097/ MCO.0000000000000480)

Cao LL, Du GH \& Wang MW 2005 Effect of salidroside on mitochondria injury induced by sodium azide. Yao Хue Xue Bao 40 700-704)

Chen H, Detmer SA, Ewald AJ, Griffin EE, Fraser SE \& Chan DC 2003 Mitofusins Mfn1 and Mfn2 coordinately regulate mitochondrial fusion and are essential for embryonic development. Journal of Cell Biology 160 189-200. (https://doi.org/10.1083/jcb.200211046)

Cheng J, Wang F, Yu DF, Wu PF \& Chen JG 2011 The cytotoxic mechanism of malondialdehyde and protective effect of carnosine via protein cross-linking/mitochondrial dysfunction/reactive oxygen species/MAPK pathway in neurons. European Journal of Pharmacology 650 184-194. (https://doi.org/10.1016/i.ejphar.2010.09.033)

Di Meo S, Iossa S \& Venditti P 2017 Skeletal muscle insulin resistance: role of mitochondria and other ROS sources. Journal of Endocrinology 233 R15-R42. (https://doi.org/10.1530/JOE-16-0598)

Dun Y, Liu S, Zhang W, Xie M \& Qiu L 2017 Exercise combined with Rhodiola sacra supplementation improves exercise capacity and ameliorates exhaustive exercise-induced muscle damage through enhancement of mitochondrial quality control. Oxidative Medicine and Cellular Longevity 2017 8024857. (https://doi. org $/ 10.1155 / 2017 / 8024857)$

Eckel RH, Grundy SM \& Zimmet PZ 2005 The metabolic syndrome. Lancet 365 1415-1428. (https://doi.org/10.1016/S0140-6736(05)66378-7)

Gowans GJ \& Hardie DG 2014 AMPK: a cellular energy sensor primarily regulated by AMP. Biochemical Society Transactions 42 71-75. (https:// doi.org/10.1042/BST20130244)

Hall DT, Griss T, Ma JF, Sanchez BJ, Sadek J, Tremblay AMK, Mubaid S, Omer A, Ford RJ, Bedard N, et al. 2018 The AMPK agonist 5-aminoimidazole-4-carboxamide ribonucleotide (AICAR), but not metformin, prevents inflammation-associated cachectic muscle wasting. EMBO Molecular Medicine 10 e8307. (https://doi. org/10.15252/emmm.201708307)

Hamacher-Brady A \& Brady NR 2016 Mitophagy programs: mechanisms and physiological implications of mitochondrial targeting by autophagy. Cellular and Molecular Life Sciences 73 775-795. (https:// doi.org/10.1007/s00018-015-2087-8)

He Y, Zhou L, Fan Z, Liu S \& Fang W 2018 Palmitic acid, but not highglucose, induced myocardial apoptosis is alleviated by Nacetylcysteine due to attenuated mitochondrial-derived ROS accumulation-induced endoplasmic reticulum stress. Cell Death and Disease 9 568. (https:// doi.org/10.1038/s41419-018-0593-y)

Herzig S \& Shaw RJ 2018 AMPK: guardian of metabolism and mitochondrial homeostasis. Nature Reviews Molecular Cell Biology 19 121-135. (https://doi.org/10.1038/nrm.2017.95)

Holley AK, Bakthavatchalu V, Velez-Roman JM \& St Clair DK 2011 Manganese superoxide dismutase: guardian of the powerhouse. International Journal of Molecular Sciences 12 7114-7162. (https://doi. org/10.3390/ijms12107114)

Huang JH, He RH, Yi LZ, Xie HL, Cao DS \& Liang YZ 2013 Exploring the relationship between 5'AMP-activated protein kinase and markers related to type 2 diabetes mellitus. Talanta $1101-7$. (https://doi. org/10.1016/j.talanta.2013.03.039)

Huang J, Tian R, Yang Y, Jiang R, Dai J, Tang L \& Zhang L 2017 The SIRT1 inhibitor EX-527 suppresses mTOR activation and alleviates acute lung injury in mice with endotoxiemia. Innate Immunity 23 678-686. (https://doi.org/10.1177/1753425917733531)

Jung HS \& Lee MS 2010 Role of autophagy in diabetes and mitochondria. Annals of the New York Academy of Sciences 1201 79-83. (https://doi. org/10.1111/j.1749-6632.2010.05614.x)

Kim JA, Wei Y \& Sowers JR 2008 Role of mitochondrial dysfunction in insulin resistance. Circulation Research 102 401-414. (https://doi. org/10.1161/CIRCRESAHA.107.165472)

Krebs M \& Roden M 2004 Nutrient-induced insulin resistance in human skeletal muscle. Current Medicinal Chemistry 11 901-908. (https://doi. org/10.2174/0929867043455620)

Larsen S, Nielsen J, Hansen CN, Nielsen LB, Wibrand F, Stride N, Schroder HD, Boushel R, Helge JW, Dela F, et al. 2012 Biomarkers of mitochondrial content in skeletal muscle of healthy young human subjects. Journal of Physiology 590 3349-3360. (https://doi. org/10.1113/jphysiol.2012.230185)

Li F, Tang H, Xiao F, Gong J, Peng Y \& Meng X 2011a Protective effect of salidroside from Rhodiolae Radix on diabetes-induced oxidative stress in mice. Molecules 16 9912-9924. (https://doi.org/10.3390/ molecules16129912)

Li L, Pan R, Li R, Niemann B, Aurich AC, Chen Y \& Rohrbach S 2011b Mitochondrial biogenesis and peroxisome proliferator-activated receptor-gamma coactivator-1alpha (PGC-1alpha) deacetylation by physical activity: intact adipocytokine signaling is required. Diabetes 60 157-167. (https://doi.org/10.2337/db10-0331)

Lin HY, Weng SW, Chang YH, Su YJ, Chang CM, Tsai CJ, Shen FC, Chuang JH, Lin TK, Liou CW, et al. 2018 The causal role of mitochondrial dynamics in regulating insulin resistance in diabetes: link through mitochondrial reactive oxygen species. Oxidative Medicine and Cellular Longevity 2018 7514383. (https://doi. org/10.1155/2018/7514383)

Matthews DR, Hosker JP, Rudenski AS, Naylor BA, Treacher DF \& Turner RC 1985 Homeostasis model assessment: insulin resistance and beta-cell function from fasting plasma glucose and insulin concentrations in man. Diabetologia 28 412-419. (https://doi. org/10.1007/bf00280883)

Maurya CK, Arha D, Rai AK, Kumar SK, Pandey J, Avisetti DR, Kalivendi SV, Klip A \& Tamrakar AK 2015 NOD2 activation induces oxidative stress contributing to mitochondrial dysfunction and insulin resistance in skeletal muscle cells. Free Radical https://joe.bioscientifica.com https://doi.org/10.1530/JOE-19-0393 (c) 2020 Society for Endocrinology Published by Bioscientifica Ltd.
Printed in Great Britain 
Biology and Medicine 89 158-169. (https://doi.org/10.1016/j. freeradbiomed.2015.07.154)

Nemoto S, Fergusson MM \& Finkel T 2005 SIRT1 functionally interacts with the metabolic regulator and transcriptional coactivator PGC1 \{alpha\}. Journal of Biological Chemistry 280 16456-16460. (https:// doi.org/10.1074/jbc.M501485200)

Nishikawa T, Kukidome D, Sonoda K, Fujisawa K, Matsuhisa T, Motoshima H, Matsumura T \& Araki E 2007 Impact of mitochondrial ROS production in the pathogenesis of insulin resistance. Diabetes Research and Clinical Practice 77 (Supplement 1) S161-S164. (https:// doi.org/10.1016/j.diabres.2007.01.071)

Popkin BM 2006 Global nutrition dynamics: the world is shifting rapidly toward a diet linked with noncommunicable diseases. American Journal of Clinical Nutrition 84 289-298. (https://doi.org/10.1093/ ajcn/84.1.289)

Rains JL \& Jain SK 2011 Oxidative stress, insulin signaling, and diabetes. Free Radical Biology and Medicine 50 567-575. (https://doi. org/10.1016/j.freeradbiomed.2010.12.006)

Ren J, Pei Z, Chen X, Berg MJ, Matrougui K, Zhang QH \& Zhang Y 2019 Inhibition of CYP2E1 attenuates myocardial dysfunction in a murine model of insulin resistance through NLRP3-mediated regulation of mitophagy. Biochimica et Biophysica Acta: Molecular Basis of Disease 1865 206-217. (https://doi.org/10.1016/j.bbadis.2018.08.017)

Subauste AR \& Burant CF 2007 Role of FoxO1 in FFA-induced oxidative stress in adipocytes. American Journal of Physiology: Endocrinology and Metabolism 293 E159-E164. (https://doi.org/10.1152/ ajpendo.00629.2006)

Suliman HB \& Piantadosi CA 2016 Mitochondrial quality control as a therapeutic target. Pharmacological Reviews 68 20-48. (https://doi. org/10.1124/pr.115.011502)

Tanaka A, Cleland MM, Xu S, Narendra DP, Suen DF, Karbowski M \& Youle RJ 2010 Proteasome and p97 mediate mitophagy and degradation of mitofusins induced by Parkin. Journal of Cell Biology 191 1367-1380. (https://doi.org/10.1083/jcb.201007013)

Tiganis T 2011 Reactive oxygen species and insulin resistance: the good, the bad and the ugly. Trends in Pharmacological Sciences 32 82-89. (https://doi.org/10.1016/j.tips.2010.11.006)

Um JH, Park SJ, Kang H, Yang S, Foretz M, Mcburney MW, Kim MK, Viollet B \& Chung JH 2010 AMP-activated protein kinase-deficient mice are resistant to the metabolic effects of resveratrol. Diabetes 59 554-563. (https://doi.org/10.2337/db09-0482)

Wang Q, Zhang M, Liang B, Shirwany N, Zhu Y \& Zou MH 2011 Activation of AMP-activated protein kinase is required for berberine-induced reduction of atherosclerosis in mice: the role of uncoupling protein 2. PLoS ONE 6 e25436. (https://doi.org/10.1371/journal.pone.0025436)

Wang J, Yang X \& Zhang J 2016 Bridges between mitochondrial oxidative stress, ER stress and mTOR signaling in pancreatic beta cells. Cellular Signalling 28 1099-1104. (https://doi.org/10.1016/j. cellsig.2016.05.007)

Wang D, Sun H, Song G, Yang Y, Zou X, Han P \& Li S 2018 Resveratrol improves muscle atrophy by modulating mitochondrial quality control in STZ-induced diabetic mice. Molecular Nutrition and Food Research 62 e1700941. (https://doi.org/10.1002/mnfr.201700941)

Xie M, Jiang L, Dun Y, Zhang W \& Liu S 2019 Trimetazidine combined with exercise improves exercise capacity and anti-fatal stress ability through enhancing mitochondrial quality control. Life Sciences $\mathbf{2 2 4}$ 157-168. (https://doi.org/10.1016/j.lfs.2019.03.027)

Yan W, Zhang H, Liu P, Wang H, Liu J, Gao C, Liu Y, Lian K, Yang L, Sun L, et al. 2013 Impaired mitochondrial biogenesis due to dysfunctional adiponectin-AMPK-PGC-1alpha signaling contributing to increased vulnerability in diabetic heart. Basic Research in Cardiology 108 329. (https://doi.org/10.1007/s00395-013-0329-1)

Zhang W, Peng M, Yang Y, Xiao Z, Song B \& Lin Z 2015 Protective effects of salidroside on mitochondrial functions against exertional heat stroke-induced organ damage in the rat. Evidence-Based Complementary and Alternative Medicine 2015 504567. (https://doi. org/10.1155/2015/504567)

Zhang H, Liu B, Li T, Zhu Y, Luo G, Jiang Y, Tang F, Jian Z \& Xiao Y 2018 AMPK activation serves a critical role in mitochondria quality control via modulating mitophagy in the heart under chronic hypoxia. International Journal of Molecular Medicine 41 69-76. (https://doi. org/10.3892/ijmm.2017.3213)

Zheng T, Yang X, Wu D, Xing S, Bian F, Li W, Chi J, Bai X, Wu G, Chen $X$, et al. 2015 Salidroside ameliorates insulin resistance through activation of a mitochondria-associated AMPK/PI3K/Akt/GSK3beta pathway. British Journal of Pharmacology 172 3284-3301. (https://doi. $\operatorname{org} / 10.1111 / \mathrm{bph} .13120)$

Zheng T, Yang X, Li W, Wang Q, Chen L, Wu D, Bian F, Xing S \& Jin S 2018 Salidroside attenuates high-fat diet-induced nonalcoholic fatty liver disease via AMPK-dependent TXNIP/NLRP3 pathway. Oxidative Medicine and Cellular Longevity 2018 8597897. (https://doi. org/10.1155/2018/8597897)

Zorzano A, Liesa M \& Palacin M 2009 Mitochondrial dynamics as a bridge between mitochondrial dysfunction and insulin resistance. Archives of Physiology and Biochemistry 115 1-12. (https://doi. org/10.1080/13813450802676335)

Received in final form 25 November 2019

Accepted 3 December 2019

Accepted Manuscript published online 3 December 2019 (c) 2020 Society for Endocrinology Published by Bioscientifica Ltd. Printed in Great Britain 